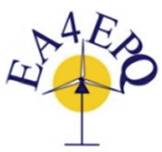

International Conference on Renewable Energies and Power Quality (ICREPQ'16)

Madrid (Spain), $4^{\text {th }}$ to $6^{\text {th }}$ May, 2016

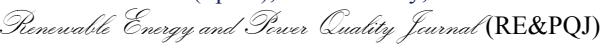

ISSN 2172-038 X, No.14 May 2016

\title{
Impact of a High Penetration of Electric Vehicles and Photovoltaic Inverters on Power Quality in an Urban Residential Grid Part II - Harmonic Distortion
}

\author{
S. Müller ${ }^{1}$, J. Meyer ${ }^{1}$, F. Möller ${ }^{1}$, M. Naumann ${ }^{1}$ and M. Radauer ${ }^{2}$ \\ ${ }^{1}$ Department of Electrical Engineering \\ Technische Universität Dresden \\ 01062 Dresden (Germany) \\ Phone number: 0049351463 35102, e-mail: jan.meyer@tu-dresden.de, sascha.mueller@tu-dresden.de \\ ${ }^{2}$ Salzburg Netz GmbH \\ Bayerhamerstraße 16, 5020 Salzburg (Austria) \\ e-mail: markus.radauer@salzburgnetz.at
}

\begin{abstract}
This paper presents a measurement-based assessment of the impact of a high penetration of plug-in electric vehicles with on-board charger (EVC) and photovoltaic inverters (PVI) on Power Quality in a public low voltage (LV) network with residential customers. This is the second part of a two-part paper. While part I provides all details about the measurement location and discusses the impact on unbalance, this part is focused on the harmonic distortion. The results are based on extensive measurements in an urban grid with almost 100 households $(\mathrm{HH}), 43$ PVIs and 36 EVCs. This paper discusses in detail the impact of PVI and EVC on selected low order harmonics as well as the total harmonic distortion at different levels of customer aggregation. At first the individual impact of a single PVI and EVC are analyzed for different operating conditions. In particular, a sunny day, a cloudy day and hours during the night are used for PVI, while periods of charging and periods of no-charging are considered for EVC. Next, the interaction between PVI, EVC and the residual household load at household level is discussed. Finally, the impact on current and voltage distortion at the level of the low voltage grid is analyzed.
\end{abstract}

\section{Key words}

Photovoltaic inverter, electric vehicle charger, power quality, harmonic distortion, harmonic phase angle

\section{Introduction}

Due to the political framework the number of EVCs and PVIs increases continuously. As inverters in PV systems as well as rectifiers in EVCs utilize power electronic circuits, they are expected to have a considerable impact on current and voltage distortion. PVIs use selfcommutating PWM-based technologies to generate a nearly sinusoidal output current. Rectifiers in EVCs usually utilize an active power factor correction in order to ensure a high energy-efficiency with good displacement factors. Both technologies are based on semiconductor switching at frequencies in the range between $10 \mathrm{kHz}$ and $100 \mathrm{kHz}$. Therefore, significant emission occurs at switching frequency, but harmonic emission below $2 \mathrm{kHz}$ is expected to be small. The emission at switching frequency is not in the scope of this paper, but further details can be found e.g. in [1]. Due to the implemented control algorithms for the semiconductor switching and the topology of the grid-side filter circuits, the harmonic emission of both technologies can significantly depends on the supply voltage waveform.

In order to perform realistic harmonic studies on the impact of high penetration of PVI and EVC in residential grids, harmonic models are required to reflect the dependency between voltage distortion and current emission with adequate accuracy. In general it can be distinguished between models in time-domain and frequency-domain. Time domain models are based on circuit models, which need a lot of information and time for development [2]. Therefore, those models are less suitable, if harmonic models of many different device types are required within a reasonable time. Frequencydomain models are often based on measurements and have different levels of complexity, depending on the objective of the analysis and the harmonic emission characteristic of the device. As the emission of most power electronic devices depends on the supply voltage distortion, simple constant current sources as used e.g. in [3] are not sufficient. Better results can be achieved by the Norton-Model (e.g. [4]), which comprises an admittance in parallel to the constant current source to account for the dependency between voltage and current of the same harmonic order. However, the coupling of harmonics of different order is not considered. For that purpose, a coupled Norton Model is needed (e.g. [5]). In order to achieve an adequate accuracy of PVI and EVC models in frequency-domain, it is recommended to use coupled Norton models as authors did in $[6,7]$.

For a comprehensive validation of the developed models, detailed measurements under real conditions are required, which can be compared with corresponding simulations. Therefore, extensive measurements have been carried out 
in an urban residential grid with a high penetration of PVIs and EVCs.

Section 2 describes the measurement framework, but only very briefly, as all details can be found in part I of the paper. The next section discusses the characteristic of an individual PVI, EVC and residual household in terms of harmonic emission followed by a section that studies the interaction between them. Finally, the total impact on the harmonic distortion in the low voltage grid is analyzed by means of voltage distortion at feeder ends and total current distortion at the LV busbar.

\section{Measurement Framework}

\section{A. Grid Details}

For the field study, an urban LV grid in the "Smart Grids Model Community Köstendorf" in Austria has been selected. With about $45 \%$ of the households having a PVI and $38 \%$ having an EVC it is a network with penetration of PVIs and EVCs well above the average. Further details about the network configuration, the data of the network elements and a single line diagram can be found in part I of the paper [8].

\section{B. Measurement Details}

Measurements have been carried out for three weeks with PQ instruments complying with the requirements of IEC 61000-4-30 class A. A one minute averaging interval has been selected and magnitude and phase angle have been recorded for all odd harmonic currents and voltages. The phase angle is measured in relation to the voltage fundamental of the respective phase as defined in IEC 61000-3-12. While magnitude is aggregated according to IEC $61000-4-30$ as RMS, the phase angle is aggregated using the phasor average method. Therefore, all individual phasors for a given aggregation interval are added vectorial and the phase angle of the resulting phasor is used as aggregated or prevailing phase angle. It should be noted that no standard definition for phase angle aggregation exists yet, but there are several activities in this direction, e.g. in IEC SC77A WG1 for an amendment of IEC 61000-4-7.

Prior the analysis, the measurement uncertainty for magnitude and phase angle of harmonic voltages and currents has been assessed. Due to the low current magnitudes at the connection point of a single household only the fundamental as well as $3^{\text {rd }}, 5^{\text {th }}$ and $7^{\text {th }}$ harmonic current are accurate enough for further analysis. If an individual measurement value of a harmonic voltage or current is lower than a predefined "accuracy threshold", it is set to zero. More details on the assessment of the accuracy threshold can be found in [9].

The analysis in this paper focuses on the first week of measurement only because the other weeks are very similar and would not lead to further findings. The figures mostly show the measurement data of that phase, to which the considered PVI or EVC was connected.

As the main objective of this study is the assessment of the impact of PVIs and EVCs on the distortion levels in the grid, only absolute values of harmonic currents are presented. Indices related to the fundamental are more suitable to characterize the equipment performance. Further details on this difference can be found e.g. in [10], where the performance of PVIs at different power conditions is discussed.

\section{Characterization of individual devices}

\section{A. PV inverter}

In order to get a first idea of the harmonic emission characteristic, a "heat map" polar plot is used to show the distribution of harmonic currents in the complex plane within the considered week. The data density is indicated by the color. Areas of high density appear in red. Low data densities are displayed in blue. Fig. 1 presents the distribution of the harmonic currents of order 3, 5 and 7 for one week. Time periods, in which the PVI is disconnected from the grid, are excluded. The prevailing phase angle is indicated by a red line. As it can be seen for the $3^{\text {rd }}$ and $7^{\text {th }}$ harmonic current, the data points are concentrated in small and distinct areas and the prevailing phase angle can be clearly identified. In contrast, the $5^{\text {th }}$ harmonic current is spread a bit more, but still within a range of $90^{\circ}$. Regarding the magnitudes, they are mostly not greater than $1 \mathrm{~A}$ with the $5^{\text {th }}$ harmonic current having the highest values.

While the polar presentation is suitable to assess potential harmonic cancellation effects, the impact of generated power or voltage distortion on the harmonic emission cannot be analyzed. Therefore, the magnitude of the harmonic currents is plotted as a function of these parameters in scatter plots (Fig. 2 and Fig. 3). A clear dependency from the generated power can be identified only in case of the $3^{\text {rd }}$ harmonic current. The other harmonics are almost randomly spread. Regarding the

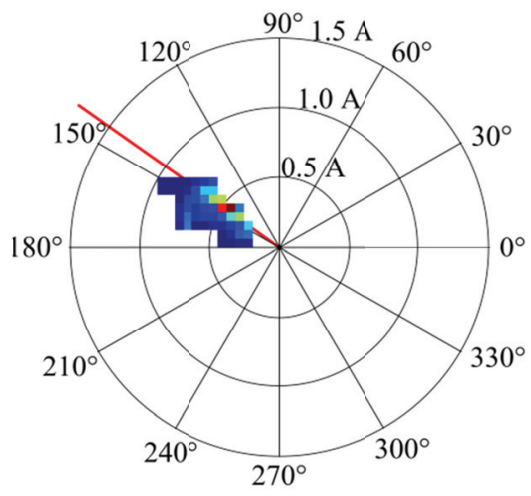

a) $3^{\text {rd }}$ harmonic current

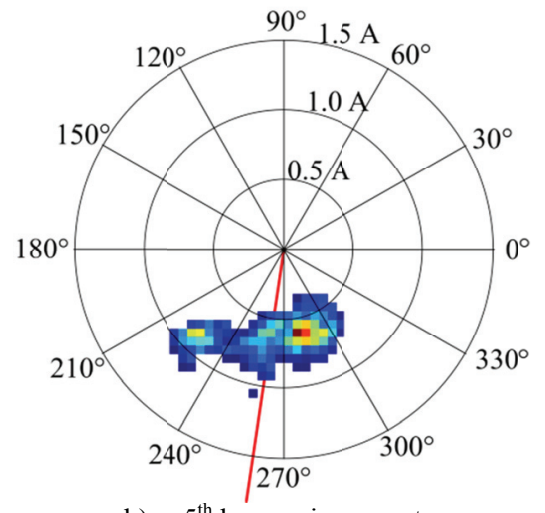

b) $5^{\text {th }}$ harmonic current

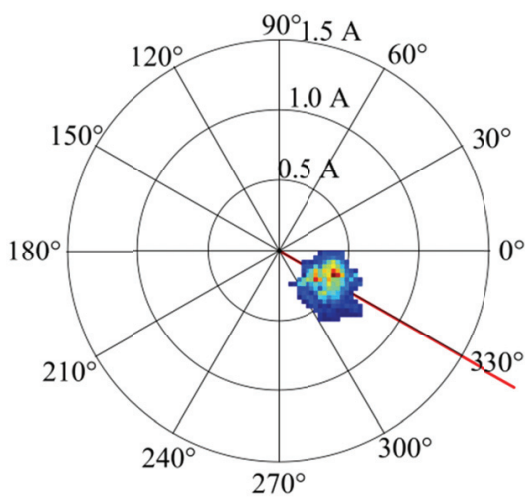

c) $7^{\text {th }}$ harmonic current

Fig. 1: Harmonic currents of an individual PVI with prevailing phase angle 


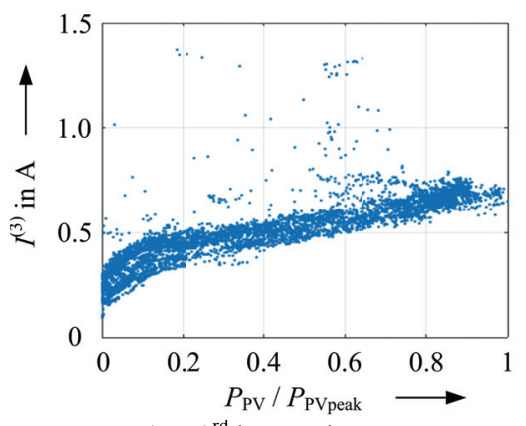

a) $3^{\text {rd }}$ harmonic current

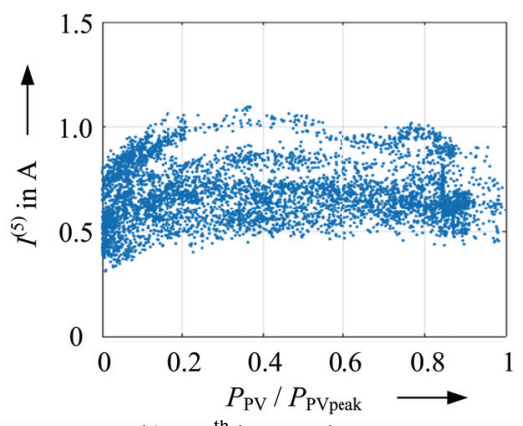

b) $5^{\text {th }}$ harmonic current

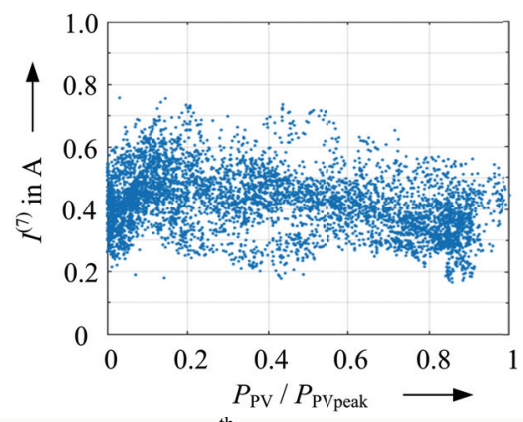

c) $7^{\text {th }}$ harmonic current

Fig. 2: Dependency of harmonic current magnitudes on generated power

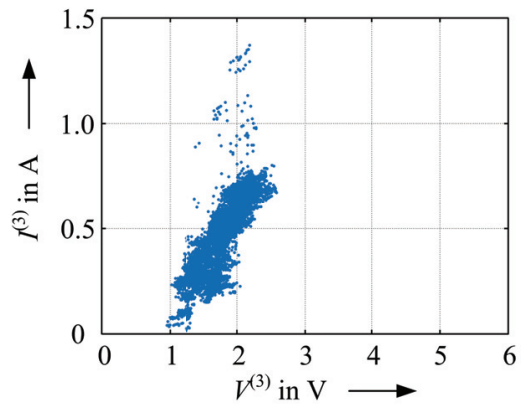

a) $3^{\text {rd }}$ harmonic current

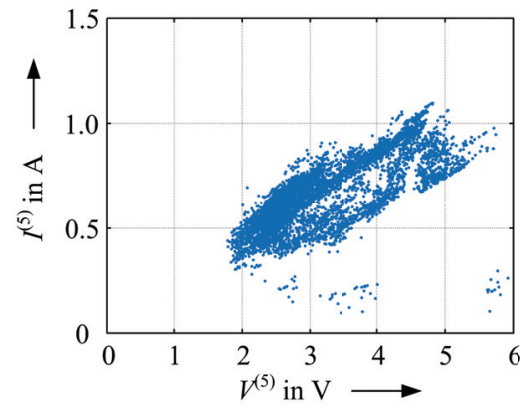

b) $5^{\text {th }}$ harmonic current

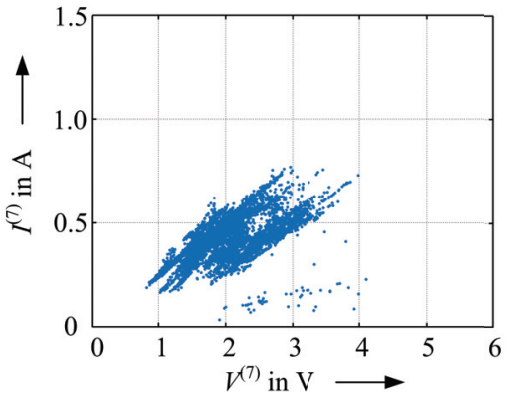

c) $7^{\text {th }}$ harmonic current

Fig. 3: Dependency of harmonic current magnitudes on their respective harmonic voltage magnitude

voltage distortion, the harmonic currents increase approximately linearly with their respective harmonic voltage, which confirms the laboratory results in [6]. This increase is most significant for the $3^{\text {rd }}$ harmonic current with about $0.5 \mathrm{~A} / \mathrm{V}$. The sensitivity for $5^{\text {th }}$ and $7^{\text {th }}$ harmonic voltage is with about $0.15 \mathrm{~A} / \mathrm{V}$ and $0.3 \mathrm{~A} / \mathrm{V}$ smaller.

\section{B. EV charger}

When considering the harmonic emission behavior of an EVC, it has to be distinguished between the three charging states: A (constant charging current); B (decrease of charging current); $\mathrm{S}$ (standby mode). Further details can be found in part I of this paper [8]. In Fig. 4 the magnitude of $3^{\text {rd }}, 5^{\text {th }}$ and $7^{\text {th }}$ harmonic currents as well as the fundamental are depicted for a full charging cycle. The fundamental serves as a reference for the charging states, which are also indicated in the figure. The most significant decrease during charging state B can be observed for the $3^{\text {rd }}$ harmonic current. In standby mode (state S) it decreases to only a quarter of the magnitude during

Fig. 4: Harmonic currents during a full charging circle (with current fundamental as reference) state A. The other harmonics are less affected. The $5^{\text {th }}$ harmonic current increases shortly in state B.

A more detailed overview is given in Table I. Besides the already discussed magnitudes, also the ranges of phase angles are summarized there. It can be seen that they can considerably change between the charging states. In particular, the phase angle of the $7^{\text {th }}$ harmonic is shifted by approximately $130^{\circ}$ between state $\mathrm{A}$ and $\mathrm{S}$.

Table I: Harmonic current ranges in different charging states

\begin{tabular}{|c|c|c|c|c|}
\hline & & $\mathbf{A}$ & B & $\mathbf{S}$ \\
\hline \multirow{2}{*}{$\boldsymbol{I}_{\mathbf{E V}}^{(3)}$} & Mag. in $\mathrm{A}$ & $0.38 \ldots 0.43$ & $0.11 \ldots 0.37$ & $0.11 \ldots 0.11$ \\
\hline & $\varphi$ in $^{\circ}$ & $117 \ldots 123$ & $-172 \ldots 164$ & $-179 \ldots-176$ \\
\hline \multirow{2}{*}{$I_{\mathbf{E V}}^{(5)}$} & Mag. in A & 0.110 .15 & $0.11 \ldots 0.22$ & $0.10 \ldots 0.11$ \\
\hline & $\varphi$ in $^{\circ}$ & $-67 . .-43$ & $-52 \ldots-38$ & $-15 \ldots-8$ \\
\hline \multirow{2}{*}{$\boldsymbol{I}_{\mathbf{E V}}^{(7)}$} & Mag. in A & $0.10 \ldots 0.15$ & $0.05 \ldots 0.12$ & $0.04 \ldots 0.05$ \\
\hline & $\varphi$ in $^{\circ}$ & $11 \ldots 27$ & $20 . .70$ & $138 \ldots 159$ \\
\hline
\end{tabular}

Similar to the PVI the harmonic emission of EVC changes with voltage distortion. However, the sensitivity is not as high as for the PVI and differs for the considered harmonic orders. While the $3^{\text {rd }}$ harmonic current does not show a clear dependency at all, the $5^{\text {th }}$ harmonic current decreases slightly with increasing $5^{\text {th }}$ harmonic voltage. In contrast, the $7^{\text {th }}$ harmonic current increases with increasing $7^{\text {th }}$ voltage harmonic. Due to the lack of space the respective figures are not presented.

\section{Households}

$3^{\text {rd }}, 5^{\text {th }}$ and $7^{\text {th }}$ harmonic current of 5 households are shown as polar plot in Fig. 5. No distinction between phases is made, as most of the equipment is single-phase. In order to analyze the emission of households individually, only that phase, where no PVI or EVC was connected or periods with disconnected PVI and EVC (e.g. parts of the night time) are considered. The 


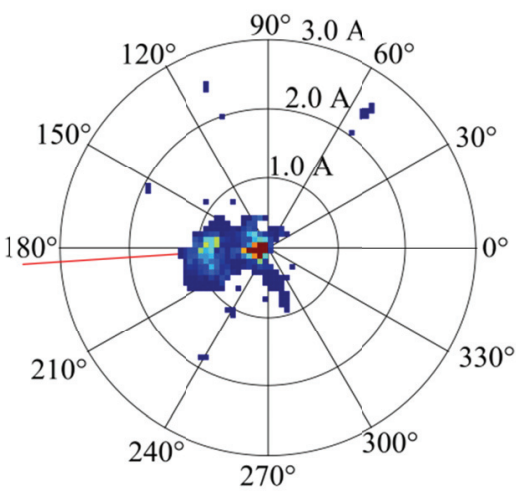

a) $3^{\text {rd }}$ harmonic current

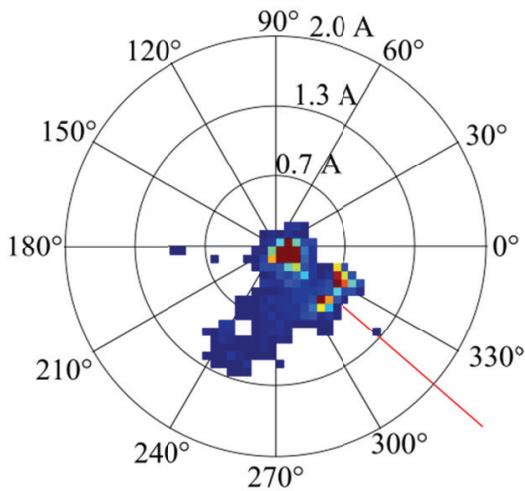

b) $5^{\text {th }}$ harmonic current

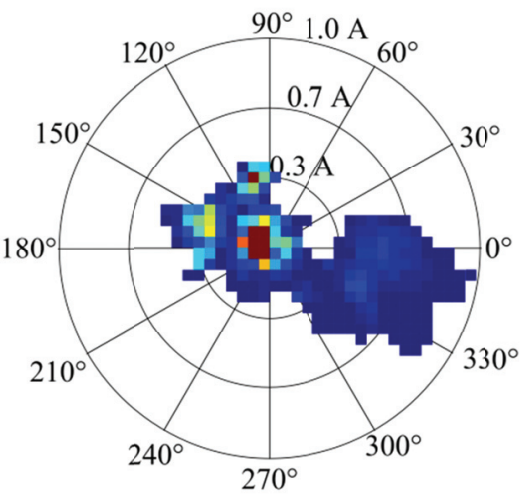

c) $7^{\text {th }}$ harmonic current

Fig. 5: Harmonic currents of individual households (without PVI and EVC) with prevailing phase angle

magnitudes are mostly below $1 \mathrm{~A}$ except for some outliers. During most of the time, the harmonic levels are very small and highest data densities can be observed in the center of the polar diagrams. Comparing the prevailing phase angles of PVI, EVC and households, for $3^{\text {rd }}$ harmonic a slight cancellation can only be expected between household and EVC, while PVI and households are almost in phase. For $5^{\text {th }}$ harmonic current a certain potential for cancellation exists between household and PVI, while EVC and household are almost in phase. In case of the $7^{\text {th }}$ harmonic no clear prevailing phase angle exists for households due to a too big dispersion and consequently, the potential for cancellation cannot be assessed. However, as diversity generally increases with harmonic order, a higher level of cancellation can be expected for higher harmonics.

\section{Harmonic interaction at household level}

In this section only short, meaningful time periods are considered.

\section{A. Household load and PV inverter}

To assess the impact of a PVI on the whole harmonic emission of the house connection, both the emission without PV generation and the emission, when the PVI generated power, are considered. The polar plots of the $3^{\text {rd }}$, $5^{\text {th }}$ and $7^{\text {th }}$ harmonic current are depicted in Fig. 6 for three different households. For the $3^{\text {rd }}$ harmonic the magnitude increases significantly by the PVI, but the phase angle remains almost constant (arithmetic addition). For the $5^{\text {th }}$ harmonic the magnitude is nearly constant, but the phase angle shifts by about $30^{\circ}$ to $50^{\circ}$, which indicates a potential of cancellation. In case of the $7^{\text {th }}$ harmonic the effect on both magnitude and phase angle is almost negligible. The resulting phase angles of all considered harmonic currents are clearly dominated by the individual PVI (cf. Fig. 1).

\section{B. Household load and EV charger}

The interaction between EVC and household is analyzed in a similar way. Fig. 7 shows the respective polar plots for three different households. In this case the three different states of the charging cycle are considered and marked in red, green and blue. The highest impact can be again observed for the $3^{\text {rd }}$ harmonic current, both in magnitude and phase angle. Magnitude increases by factor 2 and there is a phase angle shift of about $45^{\circ}$ between state A and S. In contrast, the EVC has only a marginal impact on both the magnitude and the phase angle of the $5^{\text {th }}$ harmonic current. In case of the $7^{\text {th }}$ harmonic current the phase angle of EVC is about opposite to the household load (cf. Table 1 state A and Fig. 5c), which results in rather high phase angle shift of up to $130^{\circ}$. The magnitude remains at similar, but low level, which indicates a certain "over-cancellation" of the household by the EVC. The households behave much more different for the $7^{\text {th }}$ than for the $3^{\text {rd }}$ and $5^{\text {th }}$ harmonic which indicates a higher diversity.

\section{Household load and PV inverter and EV charger}

In order to analyze the superposition of household load, PVI and EVC, a whole feeder with several PVIs and EVCs is considered. During one afternoon a time period

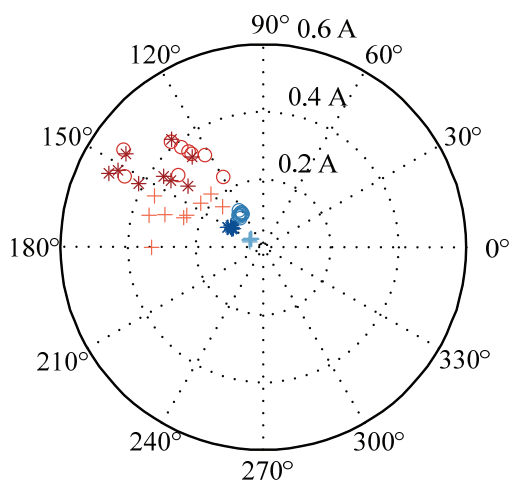

a) $3^{\text {rd }}$ harmonic current

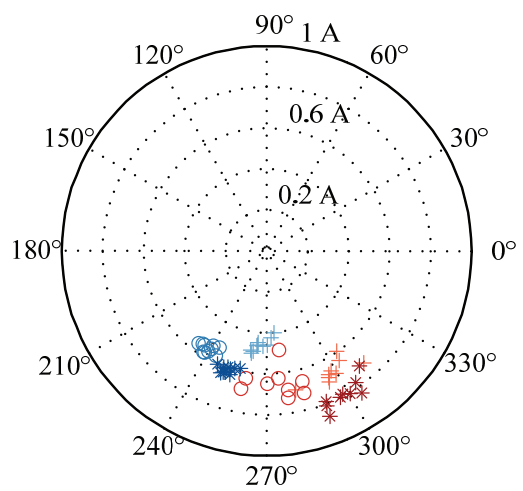

b) $5^{\text {th }}$ harmonic current

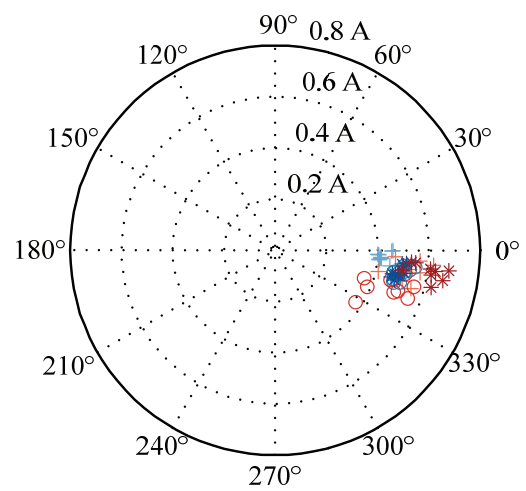

c) $7^{\text {th }}$ harmonic current

Fig. 6: Different households (different symbols) with (red) and without (blue) PV generation 


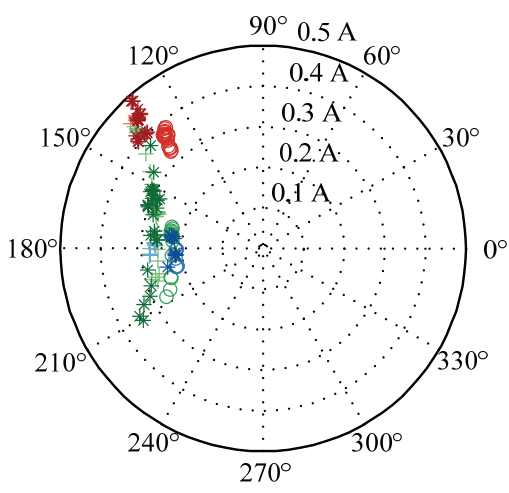

a) $3^{\text {rd }}$ harmonic current

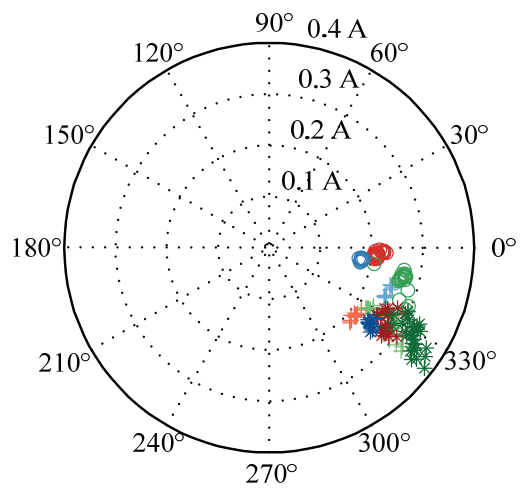

b) $5^{\text {th }}$ harmonic current

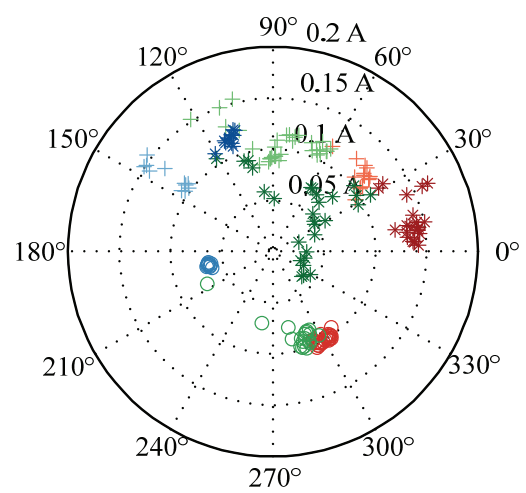

c) $7^{\text {th }}$ harmonic current

Fig. 7: Different households (different symbols) with EVC at different charging states (state A: red, state B: green, state S: blue)

was selected, where at least one EV was charging. The measured sum of the three considered harmonic currents is shown in Fig. 8. Due to the larger rated power and significantly higher coincidence factor (cf. to part I of the paper), the PVI dominates the harmonic emission compared to the EVCs. Even if a certain potential of cancellation exists, its effect on the emission would require a better balance in coincidence and number of operated devices between EVC and PVI. In case of the $7^{\text {th }}$ harmonic the direction of phase angle changes between states A and $\mathrm{S}$ differs between the households, which cannot be clearly explained at this stage, but illustrates the complexity of the interaction between different devices in low voltage grids.

\section{Impact on Harmonic Levels in the Grid}

A. Harmonic voltages at the feeder end

In general the highest voltage distortion occurs at the weaker feeder ends. The voltage at these points is much more affected by the devices connected to the considered LV grid than the harmonic voltage at the LV busbar, which is dominated (except for the $3^{\text {rd }}$ harmonic) by the background distortion of the upstream grid.

Fig. 9 shows the impact of the PVIs on the harmonic voltages at the end of feeder F1 (cf. to part I of the paper for a detailed schema) with more than $60 \mathrm{kVA}$ installed PV power and a sunny day. Except for the $3^{\text {rd }}$ harmonic voltage almost every harmonic shows a significant decrease during the daytime (operation of PVIs), when the generated power is highest. In particular the $15^{\text {th }}$ harmonic, which is close to the limit of $0.5 \%$ during the night, reduces very significantly during the time with PV generation. The reason for the increase of the $3^{\text {rd }}$ harmonic voltage is that the PVIs dominate the total $3^{\text {rd }}$ harmonic current and due to the missing background voltage from the upstream grid no potential of cancellation exists. In contrast, $5^{\text {th }}$ and $7^{\text {th }}$ harmonic voltages are transferred downstream from the MV grid and have such a phase angle that the additional harmonic voltages caused by the respective harmonic currents in the low voltage grid tend to decrease the total harmonic voltages at the feeder ends. This effect has been also observed e.g. in [11-13]. Thus, for this particular grid the combined operation of PVIs and EVCs has an overall positive impact on the harmonic voltages. The positive impact is mainly a result of the PVIs.

\section{B. Harmonic currents at the transformer busbar}

The harmonic currents of the whole grid are depicted in Fig. 10. While the magnitudes of the $3^{\text {rd }}, 5^{\text {th }}, 7^{\text {th }}$ and $11^{\text {th }}$ harmonic current increase considerably during daytime, the levels of the $9^{\text {th }}, 13^{\text {th }}$ and $15^{\text {th }}$ harmonic decrease due to possible cancellation effects. The other harmonic orders are so small that they are below the accuracy limit of the measurement devices and no conclusion can be drawn. It should be noted that a small $2^{\text {nd }}$ harmonic current exists, which increases during the day (operation of PVIs), but it is not characteristic for public LV networks. The PVIs clearly dominate the harmonic emission in this grid $\left(5^{\text {th }}\right.$ harmonic current doubles almost during the day). Even if the EVCs had also a significant harmonic current emission, their impact would be much smaller due to their significantly smaller coincidence and

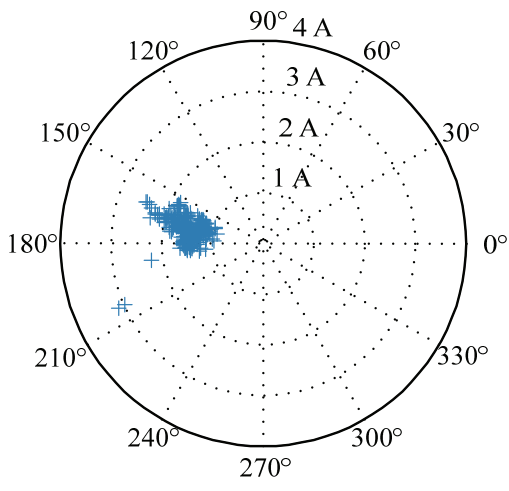

a) $3^{\text {rd }}$ harmonic current

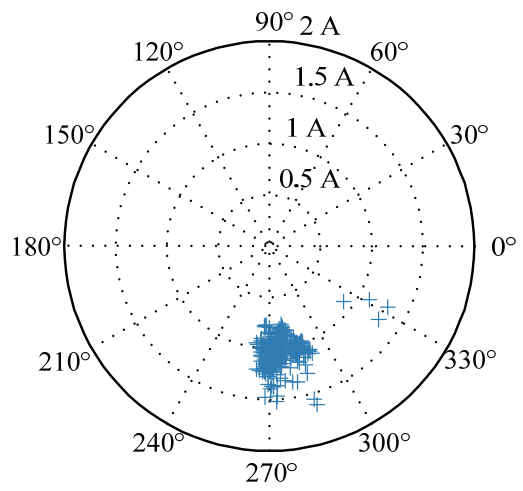

b) $5^{\text {th }}$ harmonic current

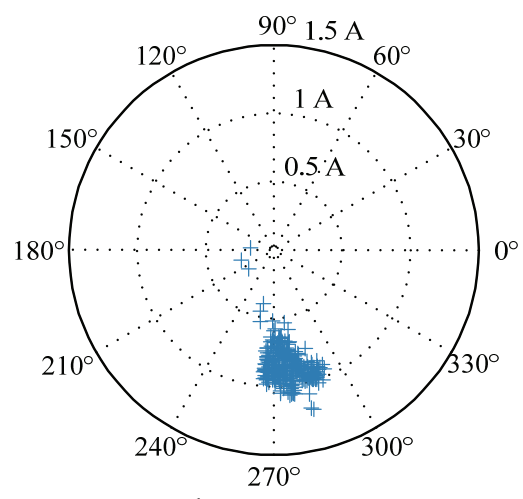

c) $7^{\text {th }}$ harmonic current

Fig. 8: Superposition of harmonic currents in one feeder with PVIs and EVCs (phase L1) 


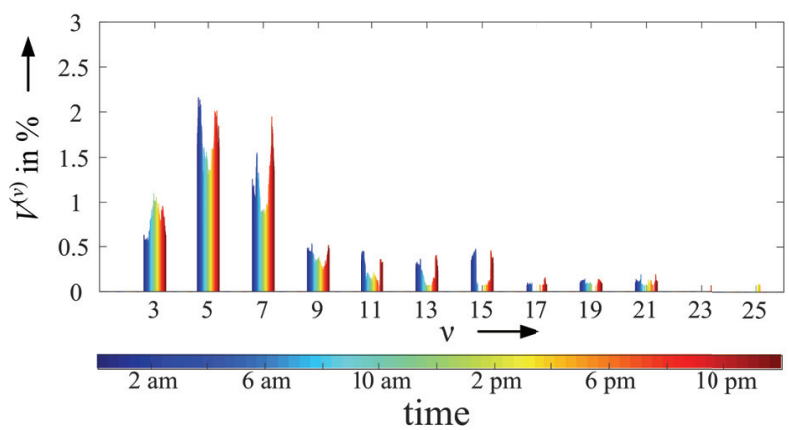

Fig. 9: Voltage distortion at the end of feeder F1 (phase L1)

therefore less parallel operating units. This is expected to be completely different in case of central charging infrastructure and larger EVC fleets.

\section{Conclusion}

Detailed measurements in a public LV grid with a high penetration of PVI and EVC have been analyzed in terms of their impact on harmonic distortion. PVIs and EVCs show a significant, not neglectable emission, particular at low harmonic orders. This is mainly caused by the existing voltage distortion and questions the current practice of testing harmonic emission at sinusoidal supply voltage. This suggests that coupled Norton models are required for accurate harmonic studies in presence of PVIs and EVCs. Due to the significant different coincidence factors of 1 for PVIs and about 0.2 for EVCs, the PVIs dominate the impact on the harmonic emission.

For the $3^{\text {rd }}$ harmonic virtually no cancellation can be expected with existing households and consequently, its total level is majorly determined by PVIs. The $5^{\text {th }}$ harmonic current is also dominated by the contribution of the PVI, however due to the different direction of the background distortion compared with the voltage caused by the PVIs, a considerable compensation effect for the $5^{\text {th }}$ harmonic voltage at the feeder ends can be observed. As most of the harmonic voltages have reduced levels during the day, a positive impact of PVIs (except for the $3^{\text {rd }}$ harmonic) can be concluded. It should be noted that in case that the prevailing harmonic voltage slowly changes during the years due to the increase of PVI penetration, the positive effect could reverse in future again. Therefore, reasonable limits for harmonic currents are indispensable.

Finally, it should be noted that due to their grid-side filter circuits both EVC and PVI frontends introduce a significant amount of capacitance into the LV grid. This results in a considerable decrease of the frequency of the first parallel resonance, which might lead to resonance related problems, which are nowadays only an issue in MV or HV networks.

The next step is to use the measurement data for validating the developed PVI and EVC models by using an appropriate simulation environment.

\section{Acknowledgement}

This work was supported by the German Federal Ministry for Environment, Nature Conservation, Building and Nuclear Safety (project ElmoNetQ, FKZ: 16EM1052) and the Salzburg Netz GmbH.

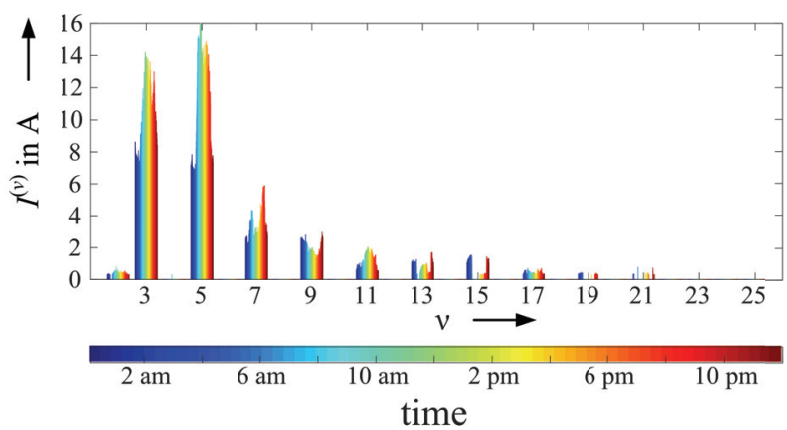

Fig. 10: Current distortion at the transformer busbar (phase L1)

\section{References}

[1] S. Schöttke, J. Meyer, P. Schegner, S. Bachmann, "Emission in the Frequency Range of $2 \mathrm{kHz}$ to $150 \mathrm{kHz}$ caused by electrical vehicle charging," EMC Europe, Dresden, Germany, 2014.

[2] A. J. Collin, S. Z. Djokic, H. F. Thomas, J. Meyer, "Modelling of Electrical Vehicle Chargers for Power System Analysis," $11^{\text {th }}$ International Conference on Electrical Power Quality and Utilisation (EPQU), Lisbon, Portugal, 2011.

[3] C. Jiang, R. Torquato, D. Salles, W. Xu, "Method to Assess the Power-Quality Impact of Plug-in Electric Vehicles," IEEE Trans. Power Delivery, vol. 29, pp. 958-965, Apr. 2014.

[4] E. Thunberg, L. Söder, "A Norton Approach to Distribution Network Modeling for Harmonic Studies," IEEE Trans. Power Delivery, vol. 14, pp. 272-277, Jan. 1999.

[5] C. F. M. Almeida, N. Kagan, "Harmonic Coupled Norton Equivalent Model for Modeling Harmonic-Producing Loads," $14^{\text {th }}$ International Conference on Harmonics and Quality of Power (ICHQP), Bergamo, Italy, Sep. 2010.

[6] S. Müller, J. Meyer, P. Schegner, "Characterization of Small Photovoltaic Inverters for Harmonic Modeling," $16^{\text {th }}$ International Conference on Harmonics and Quality of Power (ICHQP), Bucharest, Romania, 2014

[7] S. Müller, J. Meyer, P. Schegner, S. Djokic, "Harmonic Modeling of Electric Vehicle Chargers in Frequency Domain,“ International Conference on Renewable Energies and Power Quality (ICREPQ), La Coruña, Spain, 2015

[8] F. Möller, J. Meyer, M. Radauer, "Impact of a High Penetration of Electric Vehicles and Photovoltaic Inverters on Power Quality in an Urban Residential Grid Part I Unbalance," International Conference on Renewable Energies and Power Quality (ICREPQ), Madrid, Spain, 2016.

[9] T. Pfajfar, J. Meyer, P. Schegner, I. Papič, "Influence of Instrument Transformers on Harmonic Distortion Assessment," IEEE PES General Meeting, San Diego, USA, July 2012.

[10] S. Djokic, J. Meyer, F. Möller, R. Langella, A. Testa: "Impact of Operating Conditions on Harmonic and Interharmonic Emission of PV Inverters," International Workshop on Applied Measurements for Power Systems, Germany, 2015.

[11] F. Möller, S. Müller, J. Meyer, P. Schegner, C. Wald, S. Isensee, "Impact of Electric Vehicle Charging on Unbalance and Harmonic Distortion - Field Study in an Urban Residential Area," $23^{\text {rd }}$ International Conference on Electricity Distribution (CIRED), Lyon, France, 2015

[12] R. Pradatscher, R. Witzmann, G. Wirth, A. Spring, G. Becker, S. Schmidt, J. Brantl, "Untersuchungen zur Oberschwingungsbelastung in Netzen mit hoher PhotovoltaikDurchdringung," 13. Symposium Energieinnovation, Graz, Austria, 2014

[13] S. C. Vegunte, P. Twomey, D. Randles, "Impact of PV and Load Penetration on LV Network Voltage and Unbalance and Potential Solutions," $22^{\text {nd }}$ International Conference on Electricity Distribution (CIRED), Stockholm, Sweden, 2013 